\title{
Novel Means of Clinical Visual Function Testing among Glaucoma Patients, Including Virtual Reality
}

\author{
Simon E Skalicky ${ }^{1}$, George YX Kong ${ }^{2}$
}

\begin{abstract}
Computed perimetry remains the gold standard of visual field measurement among glaucoma patients. However, several emerging technologies, made possible by advances in computer programming, smartphone, tablet, or virtual reality, allow alternative means of visual function assessment. These new visual tests may one day have a useful complementary role in visual field testing and to bridge the gap between perimetry and daily experience. Many of these emerging technologies have distinct practical advantages over Ganzfield bowl-based computed perimetry. This paper outlines a discussion of some of these emerging techniques in visual function assessment in glaucoma.

Keywords: Glaucoma, Perimetry, Tablet, Virtual reality, Visual function.

Journal of Current Glaucoma Practice (2019): 10.5005/jp-journals-10078-1265
\end{abstract}

\section{INTRODUCTION}

Glaucoma is the leading cause of irreversible blindness worldwide ${ }^{1}$ and its prevalence is increasing. ${ }^{2}$ In 2015, glaucoma was responsible for 2.9 million cases of blindness in the world. ${ }^{1}$ Glaucoma, estimated to affect $3.5 \%$ of those aged $40-80$ years, is forecast to impact 76 million by 2020 and 112 million by $2040 .^{2}$ Accordingly, glaucoma poses a considerable socioeconomic burden globally. ${ }^{3}$ Key to the diagnosis and long-term management of glaucoma is serial assessment of visual function.

Patients with worsening glaucoma have increasing difficulty with light/dark adaptation, contrast discrimination, and peripheral vision-dependent activities. ${ }^{4}$ This can impact walking, driving, venturing from home, seeing at night, reading, adjusting to different levels of illumination, judging distances, and seeing peripheral objects and moving objects coming from the side, resulting in falls or motor vehicle accidents. ${ }^{5-7}$ Progressive vision impairment from glaucoma may adversely affect one's ability to perform daily activities, physical and psychological well-being, and health-related quality of life (QoL). ${ }^{3}$

Visual acuity (VA), contrast sensitivity (CS), and visual field testing by standard automated perimetry (SAP) are commonly used to quantify functional visual ability in glaucoma patients. ${ }^{8-11}$ However, they may not reflect real-world visual function. For instance, in real life, patients can be distracted, or be multitasking, and are able to move their head and use saccades to compensate for loss of peripheral vision. ${ }^{12}$ Questionnaires-also known as patientreported outcomes (PROs) - allow patients to self-evaluate their ability to perform visually related tasks, among other domains of glaucoma-related QoL. ${ }^{6}$ However, recall bias, psychological factors, and personality may influence patients' responses. Two patients with the same degree of clinically measured vision loss may rate their disability differently on a questionnaire, or may alter their responses on different days depending on mood or other factors. ${ }^{13,14}$

Many of these limitations could potentially be overcome by objective simulation of functional visual ability. Some task-specific tests have been developed and used to good effect in a research setting, and some excellent work has already been performed to evaluate falls risk, reading speed, and positional stability among glaucoma patients., ${ }^{5-22}$ Performance-based assessments of
1,2Department of Ophthalmology, Centre for Eye Research Australia, University of Melbourne, Victoria, Australia; Department of Ophthalmology, Royal Victorian Eye and Ear Hospital, Victoria, Australia Corresponding Author: Simon E Skalicky, Department of Ophthalmology, Centre for Eye Research Australia, University of Melbourne, Victoria, Australia; Department of Ophthalmology, Royal Victorian Eye and Ear Hospital, Victoria, Australia, Phone: +613 9929 8666, e-mail: seskalicky@gmail.com

How to cite this article: Skalicky SE, Kong GYX. Novel Means of Clinical Visual Function Testing among Glaucoma Patients, Including Virtual Reality. J Curr Glaucoma Pract 2019;13(3):83-87.

Source of support: Nil

Conflict of interest: Dr George Kong is Director of Glance Optical Pty Ltd

functional ability related to vision, the assessment of visual disability related to vision (ADREV) and the related compressed assessment of activities related to vision (CAARV), have been developed; however, they have technical challenges: these are physical tests that require participants to navigate a large space and outcomes may be influenced by neuro/musculoskeletal morbidities. ${ }^{4,23-25}$

With digital and smart technology that has changed the world in the past decade, there is an explosion of new opportunities to use the human/digital interface to evaluate and understand visual function for glaucoma patients. There are several emerging technologies that may one day have a useful complementary role in visual field testing and to bridge the gap between perimetry and daily experience. Whether these technologies will thrive or be quickly replaced is unclear, but they provide tantalizing glimpses of what may be possible, what may be more convenient or enjoyable to patients, and what may be more cost-effective than SAP in resource-poor healthcare settings. This article provides a brief overview of some of the emerging visual testing strategies. (For a more detailed overview of each strategy, the original sources are referenced throughout.)

\section{New Clinical Visual Tests for Glaucoma Cambridge Glaucoma Visual Function Test}

In 2013, we hypothesized that objective visual function testing could be improved using simple, reproducible computer-simulated 
activities designed to reflect daily life activities. Computer-based simulations may have a greater role in future activity limitation assessment and clinical management of glaucoma patients. Such testing may help bridge the gap in understanding between patients and clinicians as to how glaucoma may interfere with patients' daily life; it could be an important educational intervention for patients.

We designed and validated, using Rasch analysis, the Cambridge glaucoma visual function test (CGVFT), a computed objective simulation test of visual ability in patients with glaucoma that is repeatable, simple to perform, and reflective of everyday tasks. ${ }^{26}$ This comprised of 63 image-based tasks projected on a large screen, subtending $120^{\circ}$ horizontally of binocular vision (see Figs 1 and 2). The images were designed to reflect peripherally visually challenging tasks of varying difficulty levels, and patients' ability to complete the tasks was timed. Each task began with the patient looking at a central, spinning yellow star, which was a form of initial fixation to challenge their vision peripherally.

When evaluated among a cohort of 80 glaucoma patients of varying severity and 14 controls, the CGVFT had good correlation with better eye mean deviation on SAP (correlation coefficient = -0.441) as well as QoL PRO metrics such as the glaucoma activity limitation-9 questionnaire (correlation coefficient $=0.455$ ). The CGVFT was easy to administer and use but was limited in the sense that it requires projection onto a wide screen and the presence of a test invigilator. The CGVFT has recently been re-evaluated as a computer-monitor-based test (submitted for publication).

\section{Virtual Reality Glaucoma Visual Function Test}

Subsequently, a similar test was developed, this time using a three-dimensional simulation virtual reality (VR) smartphonebased headset. ${ }^{27}$ The image on the smartphone is duplicated on two halves of the smartphone, one for each viewing eyepiece, as the smartphone is inserted into the VR viewing headpiece (Fig. 3). The test was evaluated among a cohort of 93 glaucoma patients of varying severities. The user was able to "look around" the VR world, as the phone detects any head movement and alters the displayed field of view accordingly. We based our three-dimensional test of visual function using VR technology on a similar design to the CGVFT. However, this smartphone-based VR glaucoma test, the virtual reality glaucoma visual function test (VR-GVFT), was found to be not as accurate a reflection of glaucomatous visual damage as the original CGVFT. The items of this test were grouped into three categories-stationary tasks, moving ball tasks, and driving tasks.

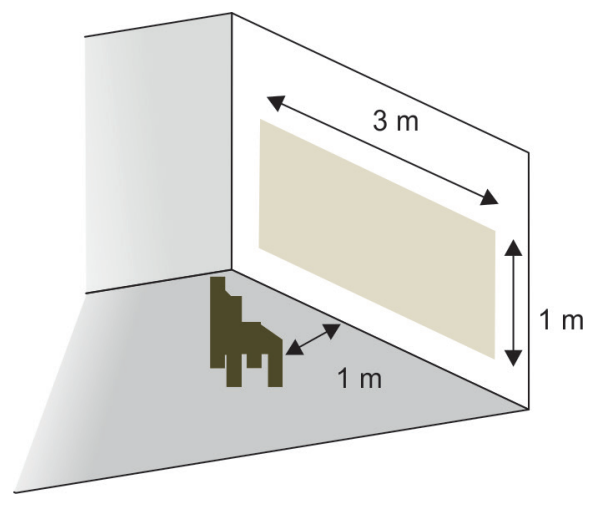

Fig. 1: Testing strategy of the original Cambridge glaucoma visual function test. Visual challenges are presented to patients projected onto a wide screen with the patients seated nearby so that $120^{\circ}$ horizontal vision is tested
Only stationary and moving ball passed Rasch analysis; and of the two only the stationary test correlated with glaucoma severity, albeit weakly ( $R=0.244 ; p=0.018$ ). Why the VR test was not as good as the widescreen projector test is unclear - the widescreen projector is more similar in nature to VF testing and this may be the reason for the greater correlation. Furthermore, the widescreen CGVFT had a form of fixation control to challenge peripheral vision use, which the VR-headset test did not.

\section{Tablet-based Perimetry}

Tablet-based perimetry is one of the most promising and wellvalidated of the emerging technologies. In comparison to the tests described above, this is very similar to traditional SAP, with the exception being administered by a touch screen tablet-both the visual stimuli as well as the patient responses. The Melbourne rapid fields (MRF) (GLANCE Optical Pty Ltd., Melbourne, Australia) has been developed as a software to administer tablet-based perimetry currently available on the Apple iPad. The software uses the zippy estimation by sequential testing (ZEST) algorithm, which is an adaptive Bayesian method, for determining retinal sensitivity measures. Each eye is tested separately and with patient wearing near spectacle correction if required. Fixation is maintained with a central fixation target, which can move to the peripheries of the tablet to further test the extremities of the field. ${ }^{28}$ A computed voice prompt guides patients throughout the test and voice reminder given at regular intervals to remind patients to maintain focus on fixation target. At the end of the test, the software generates a 24-2 visual field printout map that has good reliability and similarity with Humphrey visual field (HVF) testing (Fig. 4). ${ }^{29,30}$ When independently evaluated, global indices were highly correlated between MRF and HVF: MD $r^{2}=0.80$, PSD $r^{2}=0.77, \mathrm{VFI} r^{2}=0.85$ (all $p<0.0001)$. The ROC analysis of global indices showed reasonable sensitivity/specificity with AUC values of 0.89 (MD), 0.85 (PSD), and 0.88 (VFI). The MRF retest variability was low with ICC values at 0.95 (MD and VFI) and 0.94 (PSD). ${ }^{31}$

\section{Perimetry Using Virtual Reality Goggles}

Using a VR headpiece and a smartphone system, Tsapakis et al. describe a means of perimetric visual field testing using VR glasses, a 6-inch display smartphone, and a software that evaluates the central $24^{\circ}$ of the visual field using a fast-threshold $3 \mathrm{~dB}$ step staircase algorithm. ${ }^{32}$ The 52 testing points are all on each of the two halves of the smartphone, to stimulate either eye. The software automatically locates the blind spot and accordingly adjusts the location of the test points. The display's gamma level (luminosity per pixel), contrast, and brightness can be tightly controlled for optimal testing settings. Lens rim artifact can be a problem, with manual calibration required prior to test commencing to ensure all points are visible by the user. In a small cohort of 20 eyes from 10 glaucoma patients, this test appears to produce results similar to SAP — a high correlation between this testing strategy and SAP was reported (although it is unclear which SAP index was used for correlation).

\section{Tablet Perimetry with Gaze-tracking}

In a small pilot study, Jones et al. describe "Eyecatcher," which is tablet-based perimetry combined with gaze-tracking technology. Gaze tracker is clipped onto the bottom of the tablet and participants are shown two stimuli: the fixation and target. Elegantly, this system does not require participants pushing buttons or record seeing the target - the involuntary saccade generated by the target visible in their peripheral vision is captured by the gaze tracker, and is a 

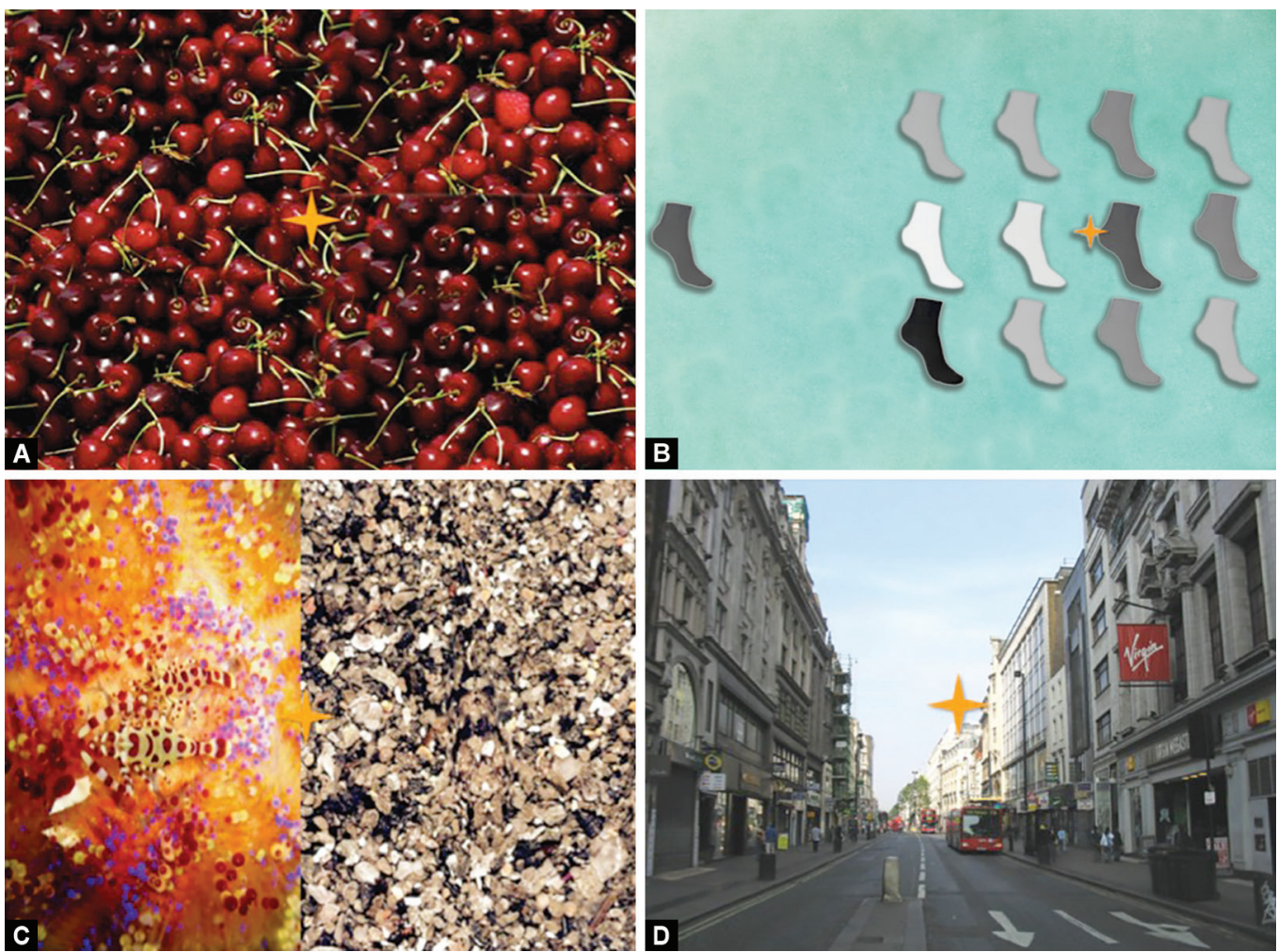

Figs 2A to D: Items from the Cambridge glaucoma visual function test (CGVFT). The CGVFT comprises timed visually challenging tasks projected onto a wide screen and designed to reflect daily living. For each task, the participant is required to begin gaze at a centrally rotating gold star and then find a target. For example, participants are asked to find a raspberry among the cherries, match a sock, identify camouflaged animals, and find items on a street scene (reproduced with permission from Skalicky SE, Lamoureux EL, Crabb DP, and Ramulu PY. Patient reported outcomes, functional assessment and utility values in glaucoma. J Glaucoma 2019;28(2):89-96)
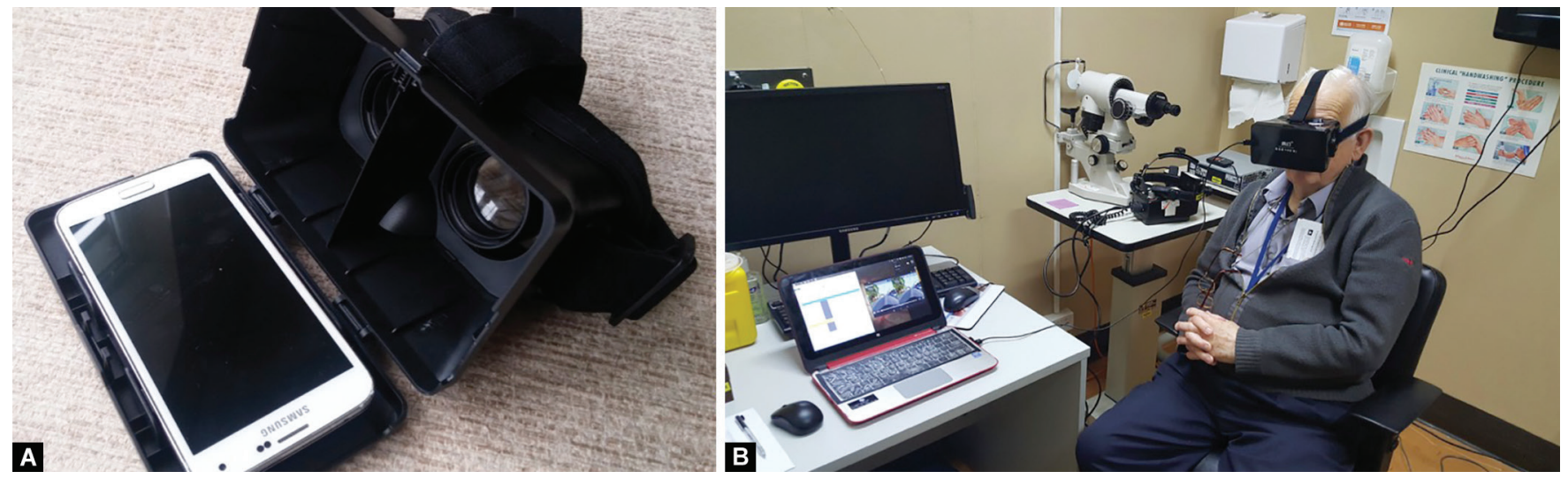

Figs 3 A and B: (A) Google Goggles viewing binocular system allowing a simulated virtual reality (VR) environment; (B) Testing strategy with patients wearing the VR headset. Their view can be tracked on the invigilators' computer screen on the desk

marker of peripheral visual registration. ${ }^{33}$ In a cohort of 12 glaucoma patients, mean Eyecatcher scores correlated with mean deviation scores $\left(r^{2}=0.64, p<0.001\right)$, and good concordance was detected between corresponding VF locations ( 84\%).

\section{Portable Multifocal Visual Evoked Potentials}

Related strategies may prove to be fruitful in detecting and monitoring visual dysfunction related to glaucoma. Nakanishi and colleagues evaluated the nGoggle (nGoggle Inc., San Diego, CA): a portable, objective brain-computer interface device integrating wireless electroencephalogram (EEG) and electrooculogram (EOG) systems and a head-mounted display (Fig. 5). ${ }^{34}$

In a case control study involving 33 participants with glaucoma and 17 healthy controls, the nGoggle demonstrated good agreement with SAP. The device was able to distinguish glaucomatous eyes from healthy eyes. The receiver operating characteristic curve area for the visual-evoked potentials parameter was 0.92 (95\% Cl, 0.86-0.96), which was larger than for SAP mean deviation (area under the curve, $0.81 ; 95 \% \mathrm{Cl}, 0.72-0.90$ ). 

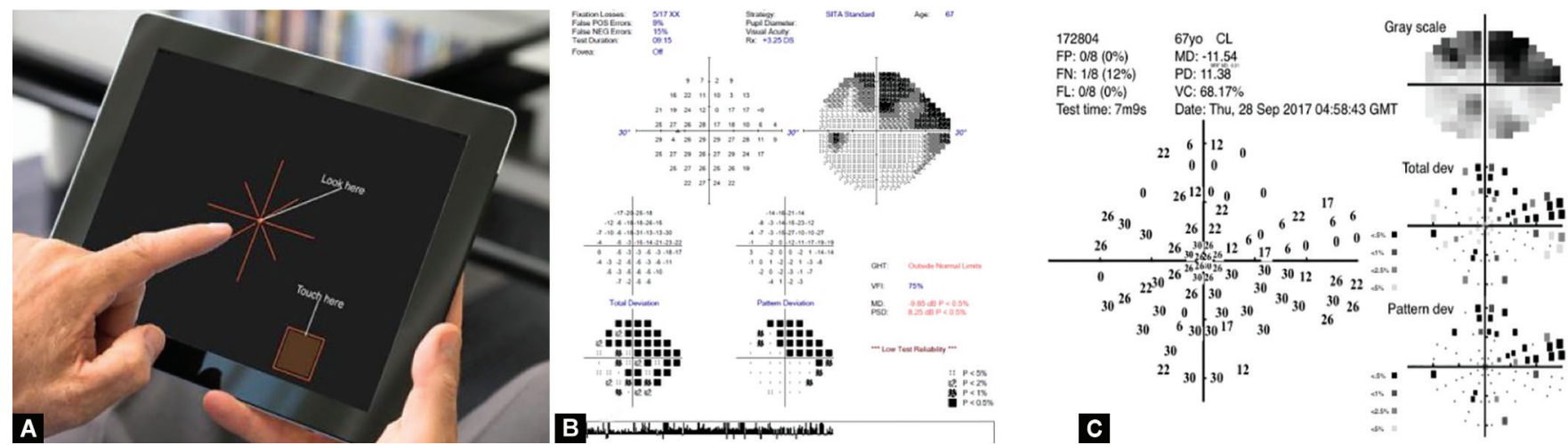

Figs 4A to C:Tablet-based perimetry. (A) User views stimuli, fixation on a tablet and presses the tablet in response to visual stimuli; (B) Humphrey visual field printout; (C) Melbourne rapid fields printout (generated by tablet perimetry) for the same patient

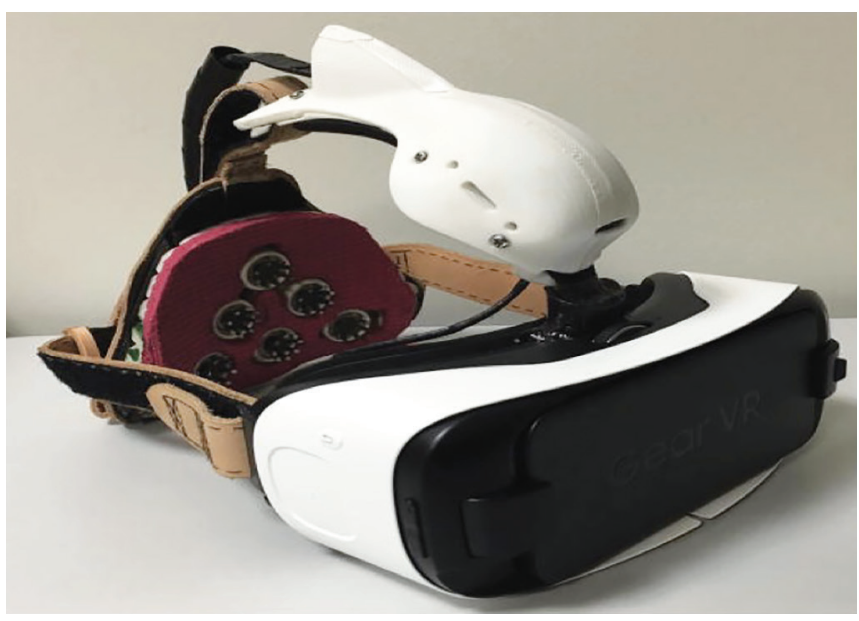

Fig. 5: Portable brain-computer interface for objective assessment of visual function loss (Nakashini et al.)

No statistically significant differences were seen for the sectoral measurements between the nGoggle and SAP.

\section{Robot Assistants for Visual Field Testing}

While the above studies looked at new technologies that could replace traditional perimetry, in a recent study McKendrick and colleagues examined whether a state-of-the-art humanoid robot is able to improve visual field testing experience by patients performing traditional SAP. ${ }^{35}$ Patients were asked to perform visual field testing four times under different feedback conditions: (1) optometrist providing feedback, (2) humanoid robot feedback, (3) computer speaker, and (4) no feedback, in random order. While the study showed no significant difference in the overall test result under all four conditions, it showed for both young and older patients there was equal preference for an optometrist or humanoid robot providing feedback and the condition of no feedback was least preferred. Robot assistants in the future may provide greater patient-centric engagement in visual field assessment, which can improve adherence and positive outcomes.

\section{Conclusion}

These testing strategies may be a passing academic interest or may represent the beginning of a new era of visual function testing for glaucoma patients. While SAP has long been a reliable, reproducible, and accurate measure of visual dysfunction due to glaucoma (and other conditions affecting the visual field), it has its drawbacks, chiefly regarding access to specialized machinery in an under-resourced healthcare setting. Tablets, smartphones, and computers, in comparison, are versatile and more or less ubiquitous.

Even in developed nations with mature health services, 50\% of glaucoma remains undiagnosed, largely because affected individuals fail to present to optometrists for routine screening. Furthermore, with health budgetary constraints it is becoming increasingly difficult to provide long-term monitoring for known glaucoma patients in healthcare institutes. There is an obvious public health advantage to being able to detect and monitor glaucoma in patients' homes using their own computers and devices.

Tests of activity limitation like the CGVFT, ADREV, and CAARV are important additions to conventional clinical assessment. They provide a critical understanding of daily patient function of glaucoma patients, allowing clinicians, patients, and policy makers to better understand the impact of glaucoma on daily life. Understanding the daily challenges of glaucoma patients, and giving patients the opportunity to demonstrate these challenges, can only be good for wholistic patient care, and lead to more meaningful clinical relationships.

Virtual reality has become highly successful and commonplace in the gaming world; however, if our experience with the VR-GVFT is replicated, it is unclear if it will have a meaningful role in disease monitoring. It is also unclear if any of this new technology can detect glaucoma earlier than SAP.

While the future of technology is always uncertain, it is hoped that innovation will continue to drive improvements and greater equitability in delivery of clinical care for people with glaucoma, as well as earlier and increased rates of glaucoma detection.

\section{References}

1. Flaxman SR, Bourne RRA, ResnikoffS, et al. Global causes of blindness and distance vision impairment 1990-2020: a systematic review and meta-analysis. Lancet Glob Health 2017;5(12):e1221-e1234. DOI: 10.1016/S2214-109X(17)30393-5.

2. Tham YC, Li X, Wong TY, et al. Global prevalence of glaucoma and projections of glaucoma burden through 2040: a systematic review and meta-analysis. Ophthalmology 2014;121(11):2081-2090. DOI: 10.1016/j.ophtha.2014.05.013.

3. Varma R, Lee PP, Goldberg I, et al. An assessment of the health and economic burdens of glaucoma. Am J Ophthalmol 2011;152(4): 515-522. DOI: 10.1016/j.ajo.2011.06.004. 
4. Lorenzana L, Lankaranian D, Dugar J, et al. A new method of assessing ability to perform activities of daily living: design, methods and baseline data. Ophthalmic Epidemiol 2009;16(2):107-114. DOI: 10.1080/09286580902738142.

5. Ramulu P, Maul E, Hochberg C, et al. Real-world assessment of physical activity in glaucoma using an accelerometer. Ophthalmology 2012;119(6):1159-1166. DOI: 10.1016/j.ophtha.2012.01.013.

6. Goldberg I, Clement $\mathrm{Cl}$, Chiang TH, et al. Assessing quality of life in patients with glaucoma using the glaucoma quality of life-15 (GQL-15) questionnaire. J Glaucoma 2009;18(1):6-12. DOI: 10.1097/ IJG.0b013e3181752c83.

7. Haymes SA, Leblanc RP, Nicolela MT, et al. Risk of falls and motor vehicle collisions in glaucoma. Invest Ophthalmol Vis Sci 2007;48(3):1149-1155. DOI: 10.1167/iovs.06-0886.

8. Burton R, Crabb DP, Smith ND, et al. Glaucoma and reading: exploring the effects of contrast lowering of text. Optom Vis Sci 2012;89(9): 1282-1287. DOI: 10.1097/OPX.0b013e3182686165.

9. Chan EW, Chiang PP, Liao J, et al. Glaucoma and associated visual acuity and field loss significantly affect glaucoma-specific psychosocial functioning. Ophthalmology 2015;122(3):494-501. DOI: 10.1016/j.ophtha.2014.09.030.

10. McKean-Cowdin R, Wang Y, Wu J, et al. Impact of visual field loss on health-related quality of life in glaucoma: the los Angeles Latino eye study. Ophthalmology 2008;115(6):941-948. DOI: 10.1016/ j.ophtha.2007.08.037.

11. Medeiros FA, Gracitelli CP, Boer ER, et al. Longitudinal changes in quality of life and rates of progressive visual field loss in glaucoma patients. Ophthalmology 2015;122(2):293-301. DOI: 10.1016/ j.ophtha.2014.08.014.

12. Burton R, Smith ND, Crabb DP. Eye movements and reading in glaucoma: observations on patients with advanced visual field loss. Graefes Arch Clin Exp Ophthalmol 2014;252(10):1621-1630. DOI: 10.1007/s00417-014-2752-x.

13. Skalicky S, Goldberg I. Depression and quality of life in patients with glaucoma: a cross-sectional analysis using the geriatric depression scale-15, assessment of function related to vision, and the glaucoma quality of life-15. J Glaucoma 2008;17(7):546-551. DOI: 10.1097/ IJG.0b013e318163bdd1.

14. Mabuchi F, Yoshimura K, Kashiwagi K, et al. High prevalence of anxiety and depression in patients with primary open-angle glaucoma. J Glaucoma 2008;17(7):552-557. DOI: 10.1097/JJG.0b013e31816299d4.

15. Crabb DP, Smith ND, Rauscher FG, et al. Exploring eye movements in patients with glaucoma when viewing a driving scene. PLoS One 2010;5(3):e9710. DOI: 10.1371/journal.pone.0009710.

16. Freeman EE, Munoz B, Rubin G, et al. Visual field loss increases the risk of falls in older adults: the Salisbury eye evaluation. Invest Ophthalmol Vis Sci 2007;48(10):4445-4450. DOI: 10.1167/iovs.07-0326.

17. Friedman DS, Freeman E, Munoz B, et al. Glaucoma and mobility performance: the Salisbury eye Evaluation Project. Ophthalmology 2007;114(12):2232-2237. DOI: 10.1016/j.ophtha.2007.02.001.

18. Glen FC, Crabb DP, Smith ND, et al. Do patients with glaucoma have difficulty recognizing faces? Invest Ophthalmol Vis Sci 2012;53(7):3629-3637. DOI: 10.1167/iovs.11-8538.

19. Ramulu P. Glaucoma and disability: which tasks are affected, and at what stage of disease? Curr Opin Ophthalmol 2009;20(2):92-98. DOI: 10.1097/ICU.0b013e32832401a9.
20. Ramulu PY, Swenor BK, Jefferys JL, et al. Difficulty with out-loud and silent reading in glaucoma. Invest Ophthalmol Vis Sci 2013;54(1): 666-672. DOI: 10.1167/iovs.12-10618.

21. Ramulu PY, van Landingham SW, Massof RW, et al. Fear of falling and visual field loss from glaucoma. Ophthalmology 2012;119(7): 1352-1358. DOI: 10.1016/j.ophtha.2012.01.037.

22. Ramulu PY, West SK, Munoz B, et al. Glaucoma and reading speed: the Salisbury eye evaluation project. Arch Ophthalmol 2009;127(1):82-87. DOI: 10.1001/archophthalmol.2008.523.

23. Altangerel $U$, Spaeth GL, Steinmann WC. Assessment of function related to vision (AFREV). Ophthalmic Epidemiol 2006;13(1):67-80. DOI: 10.1080/09286580500428500.

24. Richman J, Lorenzana LL, Lankaranian D, et al. Importance of visual acuity and contrast sensitivity in patients with glaucoma. Arch Ophthalmol 2010;128(12):1576-1582. DOI: 10.1001/ archophthalmol.2010.275.

25. Wei H, Sawchyn AK, Myers JS, et al. A clinical method to assess the effect of visual loss on the ability to perform activities of daily living. Br J Ophthalmol 2012;96(5):735-741. DOI: 10.1136/ bjophthalmol-2011-300093.

26. Skalicky SE, McAlinden C, Khatib T, et al. Activity limitation in glaucoma: objective assessment by the Cambridge glaucoma visual function test. Invest Ophthalmol Vis Sci 2016;57(14):6158-6166. DOI: 10.1167/iovs.16-19458.

27. Goh RLZ, Kong YXG, McAlinden C, et al. Objective assessment of activity limitation in glaucoma with smartphone virtual reality goggles: a pilot study. TransI Vis Sci Technol 2018;7(1):10. DOI: 10.1167/ tvst.7.1.10.

28. Vingrys AJ, Healey JK, Liew S, et al. Validation of a tablet as a tangent perimeter. Transl Vis Sci Technol 2016;5(4):3. DOI: 10.1167/tvst. 5.4.3.

29. Kong $Y X, H e M$, Crowston JG, et al. A comparison of perimetric results from a tablet perimeter and humphrey field analyzer in glaucoma patients. TransI Vis Sci Technol 2016;5(6):2. DOI: 10.1167/tvst. 5.6.2.

30. Prea SM, Kong $Y X G$, Mehta A, et al. Six-month longitudinal comparison of a portable tablet perimeter with the Humphrey field analyzer. Am J Ophthalmol 2018;190:9-16. DOI: 10.1016/j.ajo.2018.03.009.

31. Schulz AM, Graham EC, You Y, et al. Performance of iPad-based threshold perimetry in glaucoma and controls. Clin Exp Ophthalmol 2018;46(4):346-355. DOI: 10.1111/ceo.13082.

32. Tsapakis S, Papaconstantinou D, Diagourtas A, et al. Visual field examination method using virtual reality glasses compared with the Humphrey perimeter. Clin Ophthalmol 2017;11:1431-1443. DOI: 10.2147/OPTH.S131160.

33. Jones PR, Smith ND, Bi W, et al. Portable perimetry using eye-tracking on a tablet computer-a feasibility assessment. TransI Vis Sci Technol 2019;8(1):17. DOI: 10.1167/tvst.8.1.17.

34. Nakanishi M, Wang YT, Jung TP, et al. Detecting glaucoma with a portable brain-computer interface for objective assessment of visual function loss. JAMA Ophthalmol 2017;135(6):550-557. DOI: 10.1001/ jamaophthalmol.2017.0738.

35. McKendrick AM, Zeman A, Liu P, et al. Robot assistants for perimetry: a study of patient experience and performance. TransI Vis Sci Technol 2019;8(3):59. DOI: 10.1167/tvst.8.3.59. 